\title{
A Survey on Bandwidth Guaranteed Routing Techniques in Wireless Mesh Networks
}

\author{
Jobin S Thomas, \\ School Of Computer Science, \\ Karunya University, \\ Coimbatore, India
}

\author{
Noufidali V M \\ School Of Computer Science, \\ Karunya University, \\ Coimbatore, India
}

\author{
Felix Arokya Jose \\ School Of Computer Science, \\ Karunya University, \\ Coimbatore, India
}

\begin{abstract}
A Wireless Mesh Network is defined as an infrastructure network working with in as ad hoc mode.In a multi-hop wireless mesh network, it is always preferable to choose a path with higher throughput between a pair of source /destination nodes to fully exploit the network capacity. Since Wireless Mesh Networks have emerged as a practical solution for the wireless extension of the broadband internet, finding high throughput path is important. This paper surveys the different routing approaches for wireless mesh network that using bandwidth as routing metric. It discusses the problem statement, approach and the result obtaining on these methods.
\end{abstract}

\section{Keywords}

WMN, ASWP, MHEB, CAB, interference clique

\section{INTRODUCTION}

Wireless mesh networks(WMNs) have emerged as the efficient internet technology in public and private outdoor installations. Since WMN can be used as broadband wireless access technology, it can serve millions of users. Therefore cities can be expand with use of mesh networks from publicservice along with public broadband wireless access. WMN can be used in large academic and corporate campuses, municipalities, city downtown areas, and, also in residential complexes.Wireless mesh networks have attracted wide attention due to their desirable features, including selforganization, self-configuration, easy maintenance, reliable service coverage, and broadband access especially, when multiple channels or radio interfaces are applied. In a multi-hop $\mathrm{WMN}$, it is always preferable to choose a path with higher throughput between a pair of source/destination nodes to fully exploit the network capacity [5].

A wireless mesh network consists of mesh routers and mesh clients, commonly called mesh nodes that form the backbone of the network. To maintain mesh connectivity the nodes can configure automatically and re-configure dynamically. Therefore the mesh network hasself-forming and self-healing characteristics. This removes the need for centralized management[13].WMN operates with stationary mesh routers. End users can connect to these routers, which is act as backbone. Multi-hop routing is widely used in almost all wireless mesh routing techniques.

Due to interference among links, bandwidth, is a bottleneck metric in wireless networks. QoS routing in multi-hop wireless networks is very challenging due to interference among different transmissions. Interference occurs in both single channel and multi-channel wireless network.In a multi-channel wireless network, interference exists due two transmissions interfere with each other if they are using the same channel. A QoS routing seeks a source to destination route with requested bandwidth. If no such route can be found, the connection request should be blocked. In order to solve the QoS routing problem in multihop wireless networks, we need to consider two types of interference: inter-flow interference and intra flowinterference[7].

WMN backbone routers use multi-hop communication similarlyto ad hoc networks. Mobileusers connect to the backbone via stationary mesh routers. Mesh routers that play therole of access points as shown in fig.1.Ad-hoc network normally uses hop count as metric, because of user mobility new paths must be found rapidly in ad-hoc networks[8].

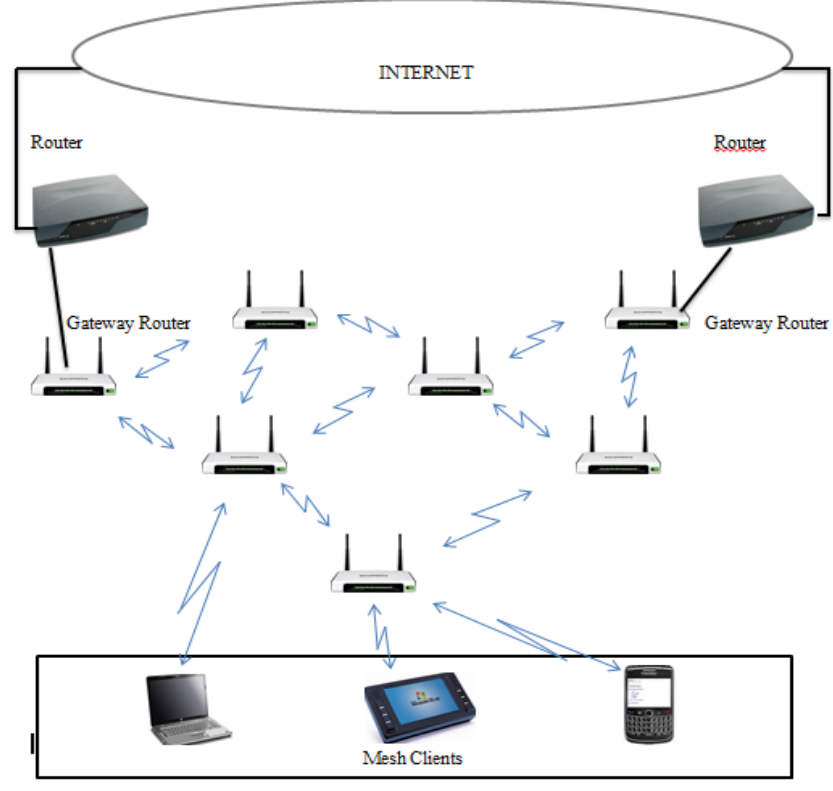

Fig: 1 wireless mesh backbone structure

\subsection{Interference Relationship}

Bandwidth based routing algorithms include the Widest Shortest Path (WSP) algorithm and the Shortest Widest Path (SWP) algorithm [10]. Both WSP and SWP cannot apply to the wireless networks because the bandwidth model in wireless environment involves interference between neighboring links [1]. Wireless transmission interference is the challenging problem in wireless networks. Then the techniques for finding widest path between source and destination will not give accurate results.

An end-to-end path mayconsist of both multi-radio channels and single-radio channels. Different channels do not interfere with each other but interference normally exists in the same channel. Given a path $\mathrm{p}=\left\langle v_{1}, v_{2}, \ldots, v_{h}\right\rangle, \mathrm{B}(1)$ denote as the available bandwidth of link 1 . Let $Q_{p}$ is the set of the maximal 
cliques containing only the links on p. If two links on a path interfere with each other, all the links between themalong the path conflict with each other [10]. The available bandwidthof path $\mathrm{p}$ is estimated as

$$
\begin{aligned}
\mathrm{B}(\mathrm{p})=\min _{q \in Q_{p}} C_{q} \\
C_{q}=\frac{1}{\sum_{l \in q}} \frac{1}{B(l)}(2)
\end{aligned}
$$

Where $C_{q}$ the bandwidth is availableover the clique q. Figure 2 illustratesan example of computation of the available path throughput interms of interference[10]. Let $\mathrm{B}(1), \mathrm{B}(2), \mathrm{B}(3)$, and $\mathrm{B}(4)$ of the network be $50,100,25$, and 20 Mbps respectively. There are two maximal cliques on path $<\mathrm{a}$; $\mathrm{b}$; $\mathrm{c}$; ; e>and they are $\{1,2,3\}$ and $\{2,3,4\}$. According toequationin

(2), $C_{\{1,2,3\}}=\left(\frac{1}{50}+\frac{1}{100}+\frac{1}{25}\right)^{-1}=\frac{100}{7}$ and $C_{\{23,4\}}=$ $\left(\frac{1}{100}+\frac{1}{25}+\frac{1}{20}\right)^{-1}=10$.According to (1), estimated availableband- width of path $\langle\mathrm{a}, \mathrm{b}, \mathrm{c}, \mathrm{d}, \mathrm{e}\rangle$ is $\min \left\{\frac{100}{7}, 10\right\}=10 \mathrm{Mbps}$.

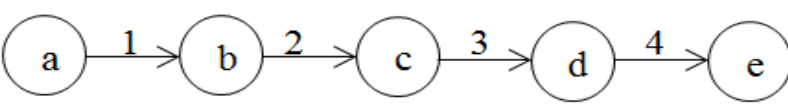

(a)

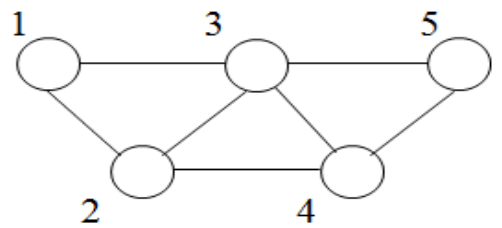

(b)

Fig .2. Clique-based interference model (a) The path (b) The conflict graph.

\subsection{Routing Metrics}

The routing metrics plays an important role in designing a routing protocol. A good routing metric [11] should find paths with links that have low loss ratio, high data rate and low levels of interference. An overview of the various routing metric for multi-hop wireless mesh networks is given as follows.

\subsubsection{Hop Count}

Hop count is used in almost all ad-hoc networks as a routing metric, especially in common routing protocols like AODV, DSR,and DSDV. This metric considers all links in the network is alike and finds the shortest path in terms of number of hops. The major disadvantage is it does not account for data rate and interference produced by the links [11].

\subsubsection{Expected Transmission Count (ETX)}

The ETX of a link [3] is the predicted number of MAC data transmissions necessary to successfully deliver a packet over the links of a path from the sender node to the receiver node. It is estimated by proactively sending a dedicated link probe packet periodically. The ETX of a path is the summation of the ETX metrics of each link in the path. The link is better when lesser the ETX metric. The disadvantage is ETX does not consider the data rate of each link over which the packets are transmitted.

\subsubsection{Weighted Cumulative Expected Transmission Time (WCETT)}

Expected transmission time ETT is an improved version of ETX by capturing the data rate used by each link. It also considers effect of packet size along with raw data rate. Both ETX and ETT do not consider the presence of multiple channels and also ETT characterizes the expected transmission time in the absence of interference in the network. The extension of ETT, WCETT [12] considers intra-flow interference. But the limitation of WCETT is, it doesn't consider inter-flow interference.

\subsubsection{Interference Aware Routing Metric (iAWARE)}

The iAWARE[11] routing metric captures the effects of variation in link loss-ratio, differences in transmission rate as well as inter-flow and intra-flow interference.Interference ratio $I R_{i}(\mathrm{u})$ for a node $\mathrm{u}$ in a link $\mathrm{i}=(\mathrm{u}, \mathrm{v})$

$$
I R_{i}(\mathrm{u})=\frac{\operatorname{SINR}_{i}(u)}{S N R_{i}(u)}(3)
$$

Where SINR is the signal to interference and noice ratio of link i. The link metric iAWARE of a link $\mathrm{j}$ as follows

$$
i A W A R E_{j}=\frac{E T T_{j}}{I R_{j}}
$$

When $I R_{j}$ for the link $\mathrm{j}$ is 1 (no interference) then $i A W A R E_{j}$ is $E T T_{j}$ which captures the link loss ratio and packet transmission rate of the link $\mathrm{j}$.

\section{ROUTING APPROACHES}

The different routing techniques that estimates widest path isdiscussed in this section. Four techniques from four different papers is surveyed and concluded as follows.

\subsection{Ad-Hoc Shortest Widest Path (ASWP)}

Mesh network usually work as an ad-hoc mode. Therefore the technique used in ad-hoc networkis relevant to mesh network also. Computing the available bandwidth of agiven path. For a given flow requests in an ad-hoc network this technique give the formula to estimate bandwidth of a path. This applies clique based path bandwidth computation method. The paper [1] presents the Ad-Hoc Shortest Widest Path (ASWP) routing problem

\subsubsection{Ad-Hoc Shortest Widest Path Problem}

It identifies path width and also it considers path length as ametric. Since loop freeness requirements is the fundamental need of a routing protocol this technique is uses path length as second metric, in terms of hop-count.This technique guarantees to admit feasible flow requests. ASWPdefines how to estimate bandwidthof a path between the given source-destination pair.The optimal solution of ASWP can be found by solving two integerprogramming problems

- finds the maximal path width

- Finds the shortest path that achieves this width.

Finding Optimal path is the major problem in ASWP, which states that consider an optimal path from node a tonode $c$ passes through $b$, it must also be the optimal path from $a$ to $b$ and from $\mathrm{b}$ to $\mathrm{c}$.Note that both the path computation and scheduling are necessary in ad-hoc QoS routing.

\subsubsection{Algorithm that solves ASWP}

The algorithm that solves ASWP is based on Bellman-Ford architecture and the $k$-shortest-path approach. For eachnode $i$, itmaintains a set of best paths. Each element of the set forms a record, denoted by $r_{s, i}=\left(s, p_{s, i} \operatorname{width}\left(p_{s, i}\right)\right.$, len $\left.\left(p_{s, i}\right)\right)$. where $r_{s, i}$ contains the source node id $s$, the complete path $p_{s, i}$ from the source to $i$, the path width, and the path lengthin terms of hopcount.To satisfy optimality requirements $\mathrm{k}$-shortest path approach is used in this algorithm. The number of records, $\mathrm{k}$ is 
predefined and the idea is to keep multiple records for each destination. The algorithm used in [1] is as follows

Let $p_{s, j}=\left(p_{s, i}, \mathrm{j}\right)=(\mathrm{s}, \ldots, \mathrm{i}, \mathrm{j})$;

Explicit loop detection;

Compute width $\left(p_{s, j}\right)$ and len $\left(p_{s, j}\right)$;

Let $r_{s, j}=\left(\mathrm{s}, p_{s, j}, \operatorname{width}\left(p_{s, j}\right), \operatorname{len}\left(p_{s, j}\right)\right.$;

If $r_{s, j}<r_{s, i}{ }^{k}$

Replaces $r_{s, i}{ }^{k}$ by $r_{s, j}$ in $R_{s, j}$;

Sort $R_{s, j}$ in the shortest-widest order;

Mark record $r_{s, j}$ active;

end

the algorithm illustrates $R_{s, i}=\left\{r_{s, i}{ }^{1} \ldots \ldots r_{s, i}{ }^{k}\right\}$ be the $\mathrm{k}$ records at node $\mathrm{i}$.

\subsubsection{Result}

ASWP can find optimal path as $\mathrm{k}$ increases to a large value where $k_{\text {opt }}$ the minimal $\mathrm{k}$ for finding optimal solution is. This algorithm is useful to route an entire sequence of flow requests. This algorithm computes the available bandwidth of paths and finds the shortest path using k-shortest path algorithm. This paper [1] proposed a distributed algorithm to solve the ASWP problem. The simulation resultscan be evaluate the performance of the algorithm, over various values of $\mathrm{k}$ and can compare with other routing techniques.

\subsection{IdentifyingWidest Paths in terms of packet loss probability and busy time fraction}

The technique in [2] addresses the problem of identifying high throughput paths in wireless mesh networks. The concept of this technique is to characterize each link by considering packet loss probability, busy time fraction, intra-flow and interflow interferences.

\subsubsection{The Approach}

The busy time fraction $\left(f_{B}\right)$,is essential in finding maximum throughput pathswhich is sensed by the link transmitter. The estimation of busy time fraction is done per-node because it is a measurement produced due to the activities of nodes. But the estimation of packet loss probability (p) has to be done perlink.The busy time fraction and packet loss probability is estimated from the interference caused by other links outside the intended path. By using 802.11 MAC protocol operation this model predicts throughput and delay of flows. The throughput and delay of each link are the functions of average input rate, busy time fraction and packet loss probability. The metric used in this method considers intra-flow interference and inter-flow interference provided busy time and packet loss.

A two-step technique to estimate available path bandwidth is introduced.

- By considering the busy time fraction and packet lossprobabilitycalculate link capacity in the path

- The link capacities can be expressed as clique-based method. This method represents a graph called link conflict graph as shown in fig 2 to express the interference relationship between links.

The technique in [2] introduces a available bandwidth metric that can compute the path providing the highest throughput by considering interference as described in 1 A.The available bandwidth of a multi-hop flow over a path is the maximum throughput it can achieved.

\subsubsection{The Routing Protocol}

This technique uses a modified version of Dijkstra's shortest path algorithm. The routing protocol uses a modified version of
Dijkstra's shortest path algorithm for route selection. If a source node needs to send a packet to destination node, source retrieves a route from centralized database. Intermediate nodes only need to follow the specified path in packet header.The modified version of Dijkstra's shortest path algorithm to search for maximum available bandwidth is given in [2].Here the cost of a link is the available bandwidth from the source to the destination of the link. The cost of any path is defined as the maximum available bandwidth between the source and destination. The available bandwidth computation is done using the cliquebasedprocedure.

\subsubsection{Result}

Having selected the route, it will not consider the routing metric, this technique uses the model to predict the available bandwidth along the chosen route.The paper [2] proposed technique to discover high throughputpaths in 802.11 mesh network. Since it uses an accurate analytical model of 802.11 , the specialty ofthis approach is the direct computation of the end-to-endavailable bandwidth. This have also shown how to our technique within a distributed routing protocolwhich exploits local measurements performed by the nodesto effectively route newly added flows, achieving significantgains with respect to existing routing metrics.

\subsection{Multi-Hop Effective Bandwidth (MHEB) metric}

The paper[5] proposes a new path metric called multi-hop effective bandwidth(MHEB). MHEB estimates the achievable bandwidth along a path. Based on the metric MHEB this paper present a new routing protocol for wireless mesh network. MHEB characterize each link in terms of inter-flow interference and intra-flow interference. It considers space channel diversity also.

\subsubsection{The Approach}

The major constraintis that all nodes are assumed stationary and equipped with multiple radios.MHEB considers, impacts of both inter/intra-flow interference and channel diversity along a path.The achievablebandwidth under intra-flow interference (ABIRF) and achievable bandwidth under the inter-flow interference (ABITF) are the two key elements in the computation of MHEB metric. Multi-hop Effective Bandwidth (MHEB) is defined as the weighted average of ABIRFand ABITF.It exploits the channel and space diversity to calculate ABIRF and considers ETX and interference degree ratio to estimate ABITF.An interference model makes transmission from node $\mathrm{u}$ to $\mathrm{v}$ is successful if the signal to interference and noise ratio (SINR) at receiver $\mathrm{v}$ is above the pre-determined threshold value $\gamma$,

$$
\frac{P_{v}(u)}{N+\sum_{k \in v^{1}} p_{v}(k)} \geq \gamma
$$

where $N$ represents the background noise, $P v(\mathrm{u})$ is the received power at node $v$ from node $u, v^{1}$ is the set of nodes located in interference range of $v$, and $P v(k)$ is the interference power from a interfering node $k$.Interference degree ratio $I D R_{i}$ (uv) for link $\mathrm{i}$, between $\mathrm{u}$ and $\mathrm{v}$ is the utilization of the channel assigned to link $\mathrm{i}$, defined as

$$
I D R_{i}(\mathrm{uv})=\frac{\sum_{k \in v^{1}} p_{v}(k)}{P_{\max }}(6)
$$

$P_{\text {max }}$ is the maximum tolerable interference power at receiver and can be calculated by $(1) \sum_{k \in v^{1}} p_{v}(k)$ is the sum of all undesired power from other transmissions at receiver node v.If there is no interference, the ratio is 0 , it means entire bandwidth of this channel is available for link $i$. If the ratio is 1 , indicating that the channel has been fully occupied by other links. 
The achievable bandwidth at link $\mathrm{i}$ under the inter-flow interference.

$$
\mathrm{ABITF}=\left(1-I D R_{i}\right) * B_{i} / \text { ETX }_{i}
$$

where, $B i$ is the original bandwidth of link $i$, ETXi [3] denotes the expected transmission attempts for a successful transmission over link $i$.

The achievable bandwidth under intra-flow interference,

$$
\mathrm{ABIRF}=\frac{L}{\frac{L}{B_{i}}+\frac{L}{B_{j}}}=\frac{B_{i} \times B_{j}}{B_{i}+B_{j}}
$$

where, $L$ is packet length, $B i$ is effective bandwidth for the setof previous links and channel $j$ has been used in previouslinks. When two links work on different channels, they couldsend and receive packets simultaneously with different radios. The effective bandwidth for these two links is $\min \left(B_{i}, B_{j}\right)$.Then the metric multi-hop effective bandwidth

$$
\text { MHEB }=\alpha \times \min (\mathrm{ABITF})+(1-\alpha) \times \operatorname{ABIRF}(9)
$$

where $\alpha$ is a tunable parameter subject to $0 \leq \alpha \leq 1$. To calculate the metric value this setting considers the two types of interference values.

\subsubsection{Routing Protocol Design}

This technique leads to a new routing protocol and the MHEB metric is used in routing procedure. By modifying AODV protocol this routing protocol can be categorize as on-demand distance vector protocol. For every node routing table creates an additional metric field called MHEB. They need to calculate the ABITF and ABIRF to update the value of MHEB.Updating process when it receives an up-to-date RREQ or RREP.One unique IP address is assigned for each interface of node. Each forwarding node inserts in RREQ information about IDR, ETX and the operating channels. This is used to compute the path metric. The node computes the ABIRF and ABITF based on the information in RREQ. If it is a new better path, the node will update the reverse route and forward it on eachof its interface otherwise, will discard the message.

Since reverse route is recorded during transmission of RREQ message, the RREP message is unicast toward the source node. In RREP message propagation, the intermediate nodes record the forwarding route to the destination node. When the source node receives the RREP message, it will send out the data packets through that pre-recorded route. If any error happens in the reception of RREP message at source node, it will resend RREQ provided maximum number of retries.

\subsubsection{Result}

MHEB based routing can improve the throughput in a multiradio wireless mesh network compare to previous techniques like hop-count based routing and weighted cumulative ETT, WCETT based routing. MHEB propose the path experiencing the least interference from source to destination, resulting in better effective bandwidth performance. As a result, interference awareness and load-balancing can be achieved simultaneously through this technique.By using this metric the paper [5] also present a new on-demand routing protocol based on AODV. The disadvantage of this technique is, it could not guarantee optimality.

\subsection{Hopby hop Packet ForwardingMechanism}

In bandwidth computation problem, satisfying consistency requirements remains as a challenge. Therefore finding optimal path from source to destination is a difficult task. It means that if only the available bandwidth is used as the routing metric, nodes may not be find the widest path. Intermediate nodes on the widest path may not make a consistent packet forwarding decisions as source identifies [6].

Hop by hop packet forwarding technique identifying the maximum available bandwidth path where every node in this path finds its own widest path. It propose a new path weight called composite available bandwidth, CABwhich captures theavailable path bandwidth information. This hop-by-hop routing protocol based on the new path weight implements based on DSDV protocol and it satisfiesthe consistency and loop-freeness requirements. Each node takes a proper packetforwarding decision, thus packet traverses over estimated best path to destination[6].

\subsubsection{The Approach}

To calculate available bandwidth of a path interference clique method is used, as we described earlier in $1 \mathrm{~A}$.This technique proposes a new path weight called $\mathrm{CAB}$. The $\mathrm{CAB}$ captures available bandwidth a path.CAB is a quadruplet $\left(\omega_{1}(p), \omega_{2}(p), \omega_{3}(p), \omega_{4}(p)\right)$ where $\omega_{1}(p)$ is the whole bandwidth of path ' $p$ ', $\omega_{2}(p)$ is the bandwidth of first three link of path $p, \omega_{3}(p)$ is the bandwidth of first two link of path $p$ and $\omega_{4}(p)$ is the bandwidth of first link of path $p$.

It has a mechanism to compare two paths based on path weight. This path weight is used in routing table and distance table of every node in the intended path. Based on this routing table and distance table, hop-by-hop packet forwarding mechanism is developed. Since each node in the intended path has routing table and packet forwarding capability, this protocol satisfies consistency and optimality requirements.

\subsubsection{The Routing Protocol}

There are five major steps in the explanation of this routing protocol. And this protocol is based on distance vector mechanism.

- First process is path selection mechanism. It has given conditions to determine whether a path isnot worthwhile to be advertised.

- Then representation of available bandwidth information as a newpath weight $(\mathrm{CAB})$ is proposed. Comparison of two paths based on path weight has given. Here clique based method is applied to estimate the available bandwidth of a path. It convinces the optimality requirements of routing protocol.

- Table construction using new path weight is the third step in QoS routing protocol. The nodes advertises the tuple ( $\mathrm{s}$, $\mathrm{d}, \mathrm{NF}(\mathrm{p}), \mathrm{NS}(\mathrm{p}), \mathrm{NT}(\mathrm{p}), \omega(p))$ to its neighbors in a route packet. $\mathrm{s}, \mathrm{d}, \mathrm{NF}(\mathrm{p}), \mathrm{NS}(\mathrm{p}), \mathrm{NT}(\mathrm{p}), \omega(p)$ are the source, the destination, the next hop, the second hop, the third hop and $\mathrm{CAB}$ on path $\mathrm{p}$, respectively.

- Afterward, this techniquepresents hop-by-hop packet forwarding mechanism using routing table. Since $N F(p)$, $\mathrm{NS}(\mathrm{p})$ and $\mathrm{NT}(\mathrm{p})$ used in routing table, each intermediate node determines the forth next hop. But in traditional protocols determination of next hop is used.

- Route update is the final process that is implemented by eliminating the path that is not worthwhile to be advertised from routing table. Even though the nodes are static the local available bandwidth of each node will change. The route update mechanism of this protocol uses the same technique as in DSDV.

\subsubsection{Result}

This metric $\mathrm{CAB}$ have better performance for finding widest path compared to other three techniques that we specified above. The main advantageof this work is a new path weight which captures the available path bandwidth information. And 
every node in path can make consistent packet forwarding decision.

\section{CONCLUSION}

In this paper, we reviewed the main bandwidth guaranteed routing techniques in WMN. Since WMN is the infrastructure network that working as an ad-hoc mode the ASWP is also discussed which deals about widest path. All methods aim to find better throughput path from source to destination. The technique hop-by-hop packet forwarding mechanism provides a routing protocol along with optimal path. But all other techniques doesn't provide a packet forwarding mechanism. There are number of routing metrics related with wireless routing. But the metrics that deals with high throughput path is very important in WMN.

\section{ACKNOWLEDGMENTS}

We would like to thank our guide Felix Arokya Jose for his guidance and comments. This work was support in part by Karunya university M.Tech thesis submission award.

\section{REFERENCES}

[1] Z. Jia, R. Gupta, J. Walrand, and P. Varaiya, "BandwidthGuaranteed Routing for Ad-Hoc Networks with InterferenceConsideration," Proc. IEEE Symp. Computers and Comm., pp. 3-9,2005.

[2] T. Salonidis, M. Garetto, A. Saha, and E. Knightly, "Identifying High Throughput Paths in 802.11 Mesh Networks: A Model-Based Approach," Proc. IEEE Int'1 Conf. Network Protocols (ICNP '07), pp. 21-30, Oct. 2007.

[3] D. Couto, D. Aguayo, J. Bicket, and R. Morris, "A HighThroughput Path Metric for Multi-Hop Wireless Routing," Proc. ACM MobiCom, pp. 134-146, Sept. 2003

[4] Y. Yang, J. Wang, and R. Kravets. "Designing routing metrics for mesh networks." In Proceedings of WiMesh, Santa Clara, CA, Sept. 2005

[5] H. Li, Y. Cheng, and C. Zhou, "Multi-Hop Effective Bandwidth Based Routing in Multi-Radio Wireless Mesh Networks," Proc. IEEE Global Telecomm. Conf. (GlobeCom '08), pp. 1-5, Nov. 2008.
[6] RonghuiHou, King-Shan Lui, Fred Baker, and Jiandong $\mathrm{Li}$, "Hop-by-Hop Routing in Wireless MeshNetworks with Bandwidth Guarantees," IEEE Trans. on Mobile Computing, vol. 11, no. 2, pp. 264-277 feb. 2012.

[7] J. Tang, G. Xue, and W. Zhang, "Interference-Aware TopologyControl and QoS Routing in Multi-Channel Wireless MeshNetworks," Proc. ACM MobiHoc, pp. 68 77, May 2005.

[8] M. Campista, D. Passos, P. Esposito, I. Moraes, C. Albuquerque,D. Saade, M. Rubinstein, L. Costa, and O. Duarte, "Routing Metrics and Protocols for Wireless Mesh Networks," IEEE Network, vol. 22, no. 1, pp. 6-12, Jan. 2002.

[9] HongqiangZhai, Xiang Chen and Yuguang Fang "alleviating intra-flow and inter-flow contentions for reliable service in mobile ad hoc networks, "IEEE Military Communications Conference, vol. 3,pp. 1640-1646, nov. 2004.

[10] H. Zhai and Y. Fang, "Impact of Routing metrics on Path Capacity in Multirate and Multihop Wireless Ad Hoc Networks," Proc. $14^{\text {th }}$ IEEE Int'1 Conf. Network Protocols (ICNP '06), pp. 86-95, Nov. 2006.

[11] A.P. Subramanian, M.M. Buddkihot, and S. Miller, "Interference Aware Routing in Multi-Radio Wireless Mesh Networks," Proc. Second IEEE Workshop Wireless Mesh Networks (WiMesh '06), pp. 55- 63, Sept. 2006.

[12] R. Draves, J. Padhye, and B. Zill, "Routing in MultiRadio, Multi-Hop Wireless Mesh Networks,” Proc. ACM SIGCOMM, pp. 114- 128, Oct. 2004.

[13] David Johnson, KarelMatthee, Dare Sokoya,LawrenceMboweni, Ajay Makan, and HenkKotze, "Building a Rural Wireless Mesh Network, "A do-ityourself guide to planning and buildinga Freifunk based mesh network by Meraka Institute, South Africa, ver 0.8 , oct 2007. 